\title{
Illusory displacement of a moving trace with respect to the grid during oscilloscope motion
}

\author{
DONALD E. PARKER, DONALD L. WOODS, and RANDY L. TUBBS \\ Miami University, Oxford, Ohio 45056
}

\begin{abstract}
Observers report that a trace streak, which follows a sinusoidal path, moves vertically with respect to the oscilloscope's grid when an oscilloscope is oscillated in the vertical plane. The vertical component of the trace streak motion with respect to the grid is illusory. This illusion is stable across a limited range of illumination and physical motion conditions. We hypothesize that this illusion is based on the manner in which the visual system calculates the vertical location of the grid and the trace: the trace location is determined on a moment-to-moment basis, whereas the grid tends to be seen in its average vertical position. The results of two experiments indicate that this hypothesis can account, at least partially, for the illusion. The illusion may have practical implications for pilots or navigators who track target blips on radar screens in moving aircraft.
\end{abstract}

During a discussion of vertibular control of eye movements, Kabrisky (Note 1) asked us to explain a phenomenon that he had noted. We were unable to do so at the time. However, the problem was intriguing, and we recently completed a series of experiments that allows us to present a preliminary response to Kabrisky's challenge.

Kabrisky's phenomenon can be demonstrated as follows. Stand looking at an oscilloscope in a darkened room. With both the grid and trace dimly illuminated and the trace speed set about $20 \mathrm{~cm} / \mathrm{sec}$, stamp your foot as the trace passes horizontally across the screen. You may have to repeat the procedure several times to achieve the appropriate timing. The phenomenon that you should see is a transient vertical displacement (pulse, blip) of the trace. The trace frequently will appear to touch or even cross one of the oscilloscope screen's horizontal grid lines.

Kabrisky said that he initially attributed this phenomenon to loose wires in the oscilloscope; only after repeated observations in the company of his colleagues was he convinced that the vertical component of the trace motion was in the observer's head rather than on the screen.

The interesting aspect of this phenomenon is not the vertical motion of the trace per se; rather, the problem is the observer's report that the trace streak

This investigation was supported in part by Contract F $3361573 C 4002$ between Miami University and the United States Air Force. We thank A. Pantle, M. Dainoff, J. Jahnke, P. Capretta, M. Moore, and L. Johnston for their comments and help. Requests for reprints should be addressed to Donald E. Parker, Department of Psychology, Miami University, Oxford, Ohio 45056 . moves vertically with respect to the oscilloscope screen grid.

Our delay in pursuing an answer to Kabrisky's question can be partially accounted for by the physical difficulty of producing the illusion: after a few trials, our feet became sore. Pain remained a problem, but was distributed following the discovery by one of our colleagues (G. Allen) that the illusion could be produced if we hit ourselves on the head instead of stamping our feet. Our breakthrough came when we realized that an apparently analogous illusion could be produced if the observer remained stationary and the oscilloscope was displaced.

When the oscilloscope was oscillated sinusoidally in the vertical plane, both the trace streak and the oscilloscope grid appeared to move vertically. The trace streak produced a fading sinusoidal wave as it passed across the oscilloscope screen. The grid appeared to oscillate vertically or horizontally. However, observers reported the clear perception that the trace streak moved vertically with respect to the grid. This aspect of the apparent trace motion was illusory because, physically, the trace path was always the same vertical distance from the horizontal grid lines; although the trace followed a sinusoidal path in "room coordinates," it moved in a straight horizontal path with respect to the grid ("grid coordinates").

Three experiments are included in this paper. Experiment 1 demonstrated that the basic illusion could be produced under various experimental conditions employing naive subjects. Experiment 2 was designed to examine our hypothesis that the illusion was based on the manner in which the visual system calculated the vertical position of the grid. Experiment 3 examined a range of oscilloscope oscillation rates. 


\section{EXPERIMENT 1}

Experiment 1 was undertaken to demonstrate the stability of the basic illusion under a variety of stimulus conditions and to determine the reaction of unbiased, naive observers.

\section{Method}

Subjects. The subjects were eight students enrolled in the introductory psychology course at Miami University who volunteered to serve without pay. None of the subjects reported prior knowledge of the nature or hypotheses of the experiment.

Apparatus. Vertical oscillation of the oscilloscope (Tektronix Type 564) was achieved by mounting the oscilloscope on a specially constructed shake table. The shake table consisted of a board that was free to rotate around an axle. One end of the board was driven by a lever that was attached to a variable-speed dc motor, and the oscilloscope was attached to this board. Relatively smooth sinusoidal oscillation was achieved by balancing the weight on the board and by locating a fly-wheel between the dc motor and the drive lever. Amplitude of oscilloscope displacement was varied by changing the location of the drive-lever pivot on the fly wheel.

The oscilloscope motion was monitored with an accelerometer (Statham Model F6-340) mounted on the oscilloscope. Permanent records of the accelerometer output were obtained with an oscillograph (Grass Model 7). Approximately sinusoidal motion could be obtained in the frequency range between 2.0 and $3.5 \mathrm{~Hz}$. All of the observations described in this paper were obtained with a peak-to-peak oscilloscope displacement of $0.6 \mathrm{~cm}$.

Trace brightness was controlled with a 10-turn precision potentiometer, which was installed in place of the factory unit, and grid illumination was controlled with the factory-installed potentiometer.

The oscilloscope was operated in the nonstorage mode and exhibited a rapid trace decay at the trace brightness settings employed. The screen phosphor was P31.

The amplified accelerometer output was used to trigger the oscilloscope's horizontal sweep circuit. With this procedure, the phase of the waveform produced by the trace appeared the same across successive sweeps.

Procedure. The subjects were read the following instructions: "First, I am going to turn out the lights so that your eyes begin to adapt to the dark. Your task in this experiment is to look at two patterns of light and then tell me what you see. One of the patterns of light is this grid (example). The other pattern is this moving trace of green light (example). When I ask you, I would like you to tell me how these two patterns of light move in relation to each other. One of them could move and the other not move, or they both could move in either the same or different manners. I just want you to tell me what you see on the screen in front of you. Are there any questions?"

The subjects were dark adapted for $5 \mathrm{~min}$. They then viewed the oscilloscope screen in a darkened room through an opening in a curtain from a distance of $50 \mathrm{~cm}$. The oscilloscope was oscillated $0.6 \mathrm{~cm}$ (peak-to-peak) at 2.5 or $3.0 \mathrm{~Hz}$. The grid, which was the standard graticule supplied by the manufacturer, consisted of an $8 \times 10 \mathrm{~cm}$ rectangle divided into $1-\mathrm{cm}^{2}$ segments. Grid brightness was set at 0.20 or $3.82 \mathrm{~cd} / \mathrm{m}^{2}$; trace brightness was equivalent to 0.14 or $2.40 \mathrm{~cd} / \mathrm{m}^{2}$ of continuous illumination. (These illumination measurements were obtained with a Prichard photometer, Model 1980.) The trace was moved in a straight-line path from left to right across the screen by the oscilloscope's horizontal sweep circuit sawtooth function at a velocity of $25 \mathrm{~cm} / \mathrm{sec}$. Each subject responded to all eight combinations of oscilloscope oscillation rate, grid brightness, and trace brightness, which were presented in random order.

The subjects' verbal responses were retained on a tape recorder. At the termination of the stimulus presentations, the subjects drew their impressions of the motion.

\section{Results}

With the exception of three of the eight subjects, who were unable to see the low intensity $\left(0.14 \mathrm{~cd} / \mathrm{m}^{2}\right)$ trace streak during high intensity $\left(3.82 \mathrm{~cd} / \mathrm{m}^{2}\right)$ grid illumination, all stimulus combinations elicited reports of the basic illusion. The trace streak (moving dot) was reported to move in a "snake-like" manner across the oscilloscope screen, while the grid appeared to move "toward me and away from me" (five of eight subjects) or up and down. In all instances, the subjects' drawings and verbal reports indicated that the trace streak appeared to move vertically with respect to the grid.

The basic motion patterns reported by the subjects did not change as a result of changes in grid intensity, trace intensity, or oscilloscope oscillation rate. Two of the subjects changed their reports of grid motion after the second trial, from "up and down" to "toward me and away from me" for one subject and in the reverse order for the other subject. In both cases, the second type of grid motion reported was stable across the remaining six trials. The drawing for subject $D$, who reported that the grid moved back and forth while the trace streak moved up and down, is presented in Figure 1.

\section{Discussion}

The results of Experiment 1 indicate that the basic illusion that we have described is stable under a variety of stimulus conditions.

It has been suggested that the illusion which we produced by shaking the oscilloscope results from latency differences in two neural pathways. This suggestion was based on the idea that information concerning the grid and the trace is carried in separate neural pathways (perhaps analogous to $X$ and Y cell pathways - see Enroth-Cugell \& Robson, 1966) and that neural response latencies in these two pathways should vary with stimulus intensity (see Grusser \& Grüsser-Cornehls, 1973). It was suggested further

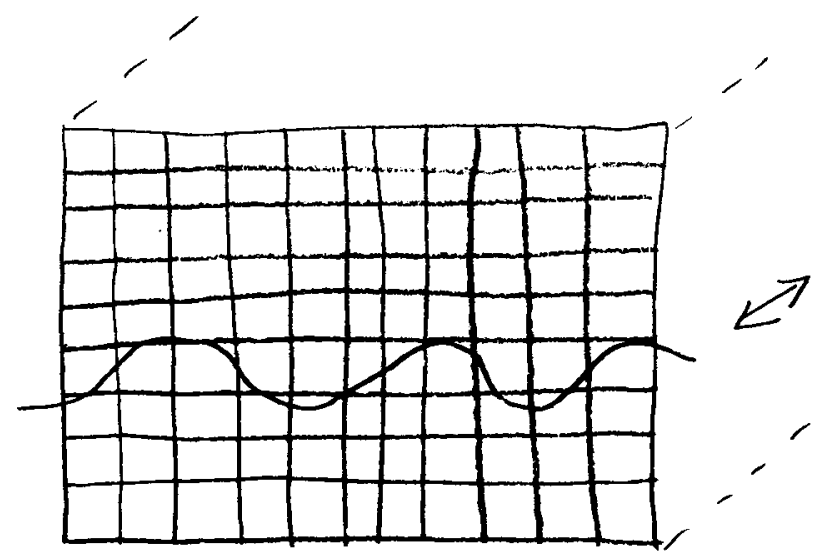

Figure 1. Drawing by subject indicating apparent trace streak and grid motion during vertical oscillation of the oscilloscope. The subject perceived the grid as moving "back and forth" and the trace as moving in a "snake-like"' fashion across the screen. 
that the relative motion of the grid and the trace should change following changes in the relative intensity of these stimulus components.

Experiment 1 indicates that the relative perceived motion of the grid and the trace did not change with variation in grid and trace brightness, over a limited range. This observation militates against the hypothesis that the illusion is dependent upon latency differences in two neural pathways.

\section{EXPERIMENT 2}

We hypothesize that the illusion described in Experiment 1 results from the manner in which the visual system calculates the apparent spatial location of the grid and the trace. The location of the trace is calculated on a moment-to-moment basis; the vertical location of the trace is continuously updated. The grid, however, tends to be located in its average position. Because the grid is continuously present, perception of the grid's vertical location is based on a longer time-sample of stimuli than the time-sample used for determining trace location. Consequently, the trace is "correctly" located (in terms of the proximal stimulus to the retina), whereas the grid location is illusory. Because the grid ordinarily is seen as "ground" and the trace as "figure," the trace is reported to move vertically with respect to the grid.

Experiment 2 was designed to examine the hypothesis. Subjects estimated the apparent "level" of the sinusoidal trace with respect to the grid under the conditions of continuous grid illumination and transient grid illumination. For the transient condition, the grid was illuminated only at particular points in the shake table vertical displacement cycle. If our hypothesis was true, the apparent "level" of the trace with respect to the grid should be the same during transient grid illumination at phase angles of 0 or $180 \mathrm{deg}$ as during continuous grid illumination.

\section{Method}

Subjects. Six students who were experienced in performing psychophysical judgments served as subjects in Experiment 2.

Apparatus. The basic apparatus for shaking the oscilloscope, monitoring oscilloscope motion, and triggering the oscilloscope's horizontal sweep circuit was the same as that described in Experiment 1. Additional apparatus to provide alternative techniques for grid illumination was as follows.

The oscilloscope grid was illuminated in two different ways. Continuous grid illumination (CGI) employed the light bulbs located under the plastic graticule, which were operated in the normal manner. Phase-locked transient grid illumination (PLTGI) was achieved with a stroboscope (General Radio Type 1531). The duration of the stroboscope flash was $3 \times 10^{-6} \mathrm{sec}$. Light was transmitted from the stroboscope to the location of the light bulbs that ordinarily illuminated the grid by fiber optic light guides that were enclosed in opaque jackets. Grid illumination intensity was $1.27 \mathrm{~cd} / \mathrm{m}^{2}$ for both CGI and PLTGI, trace illumination was equivalent to $1.90 \mathrm{~cd} / \mathrm{m}^{2}$ of continuous illumination, and the background was $0.02 \mathrm{~cd} / \mathrm{m}^{2}$.

Transient grid illumination was locked at various phase angles with respect to the oscilloscope's vertical motion. A microswitch was closed by a cam on the fly wheel and triggered one time-base of a Tektronix 535 oscilloscope. The second time-base, which activated the stroboscope, was triggered at various time delays after the first time-base had been triggered.

Procedure. Six subjects compared the apparent vertical position (level) of the trace with respect to the grid during CGI and PLTGI. An experimental session consisted of 20 trials during which all of the stimulus parameters were held constant with the exception of the phase angle between PLTGI and the oscilloscope displacement. At the beginning of each trial, the phase angle between oscilloscope displacement and PLTGI was fixed. Within an experimental session, this phase angle was varied across trials in random order. The oscilloscope was placed in motion and the subject noted the level of the trace under CGI and PLTGI. The subject's task was to estimate verbally the level of the trace during PLTGI with respect to the level of the trace under CGI. (In other words, the subjects indicated how much "higher" or "lower" the trace was during PLTGI when compared with the apparent vertical location of the trace during CGI.) The subjects used grid subdivisions as units of measurement; their estimates were ordinarily in the range of $\mathbf{2}$ to $\mathbf{3}$ grid subdivisions (approximately $0.5 \mathrm{~cm}$ ).

\section{Results}

When the oscilloscope was oscillated vertically during CGI, the trace streak appeared to follow a sinusoidal path while the grid appeared to move up and down ( $y$ axis) or back and forth ( $\mathrm{z}$ axis) over a small distance. Vertical trace displacement appeared greater than vertical grid displacement; the trace seemed to move vertically with respect to the grid as described previously. The sinusoidal trace streak was perceived to be at a particular "level" (vertical position) with respect to the grid. For example, the top of the sinusoidal trace might appear to barely touch the third grid line.

Transient (stroboscopic) illumination of the grid during vertical oscillation of the oscilloscope also resulted in perception of a sinusoidal trace that appeared to be at a particular level with respect to the grid. In this case, however, the grid appeared to be stationary. Variation of the phase angle between the stroboscopic grid illumination and the periodic oscilloscope displacement produced changes in the perceived level of the sinusoidal trace with respect to the grid.

Figure 2 illustrates observations of perceived level (vertical position) of the trace with respect to the grid during PLTGI relative to the perceived level of the trace during CGI as a function of the phase angle of the PLTGI with respect to vertical oscilloscope displacement. Positive ordinate values indicate that the trace was seen as higher during PLTGI than during CGI and negative values indicated that the trace was seen as lower during PLTGI than during CGI, for particular PLTGI-oscilloscope displacement phase angles.

The best-fitting sine function generated by these points crosses the mean ordinate value at $-8 \mathrm{deg}$ and +172 deg. The data points presented in the figure were obtained from two subjects for two experimental sessions. Data taken from the other four 


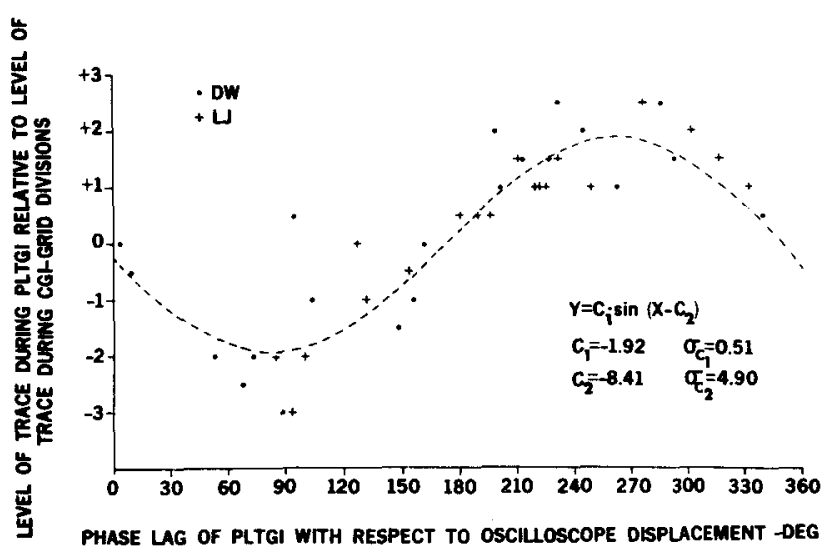

Figure 2. Perceived level (vertical position) of the trace with respect to the grid during phase-locked transient grid illumination (PLTGI) relative to the perceived level of the trace during contimuous grid illumination (CGI) as a function of the phase angle of the PLTGI with respect to vertical oscilloscope displacement.

subjects and under different experimental conditions (see Experiment 3) are essentially equivalent to those presented in Figure 2.

\section{Discussion}

The preceding observations can be interpreted as follows. For this analysis, it is assumed that the eye was stationary. First, consider the light stimulus to a stationary eye associated with traversal of a trace on a stationary oscilloscope screen. To the extent that the eye was held steady, the trace excited a linear sequence of retinal receptors (or a sequence of retinal receptors ordinarily excited by a straight line). Second, consider the light stimulus to a stationary eye associated with traversal of a trace across an oscilloscope screen when the oscilloscope was oscillated in the vertical plane. In this second case, the trace excited a sinusoidal sequence of retinal receptors (or a sequence of receptors ordinarily excited by a sinusoidal line). Therefore, the reports of a fading trace streak moving in a straight path when the oscilloscope was stationary and moving in a sinusoidal path when the oscilloscope was oscillating are readily understandable in terms of the retinal stimulus.

The third stimulus condition to be considered is that of PLTGI. As in the second case, because the oscilloscope oscillated in the vertical plane, the trace described a sinusoid on the retina. The grid was illuminated only at a particular point in the oscilloscope's vertical oscillation cycle. Since the grid was always illuminated when the oscilloscope was at the same vertical position, the grid appeared fixed to the subject; consequently, the trace appeared to move vertically with respect to the grid. Consider that the trace excited the same retinal receptors, regardless of the phase lag of the grid illumination with respect to oscilloscope motion. By changing the phase lag of the grid illumination, the retinal receptors that the grid excited were changed, essentially moving the grid up and down on the retina. Because the grid was seen as ground by the subjects, they reported that the level of the trace changed with respect to the "fixed" grid as the phase angle of PLTGI was varied.

Finally, consider the stimulus conditions produced by CGI. Under these conditions, the grid appeared slightly blurred relative to the sharp trace; but it was clear to the subjects that the trace moved vertically with respect to the grid. For oscilloscope verticaloscillation rates of $3.25 \mathrm{~Hz}$ or less, it was an easy task for the subjects to specify the apparent level of the trace with respect to the grid, and the apparent level of the trace did not change so long as the stimulus conditions were held constant. The failure of the subjects to see the grid as moving vertically over as great a distance as the trace is the basis for the illusion.

Before the observations summarized in Figure 2 were obtained, we considered two possibilities for visual system calculation of the grid position during CGI. The first possibility, which provided the hypothesis for the experiment, was that the subjects would locate the grid in the average vertical displacement position. The second possibility, which was derived from photographic records of the shaking oscilloscope, was that the subjects would locate the grid at one or the other (or both) of its peak displacement positions because at the peak of displacement the grid velocity was least and, therefore, the stimulus to the retinal receptors should have been strongest (physically, if not physiologically). Support for this second possibility would be obtained if the trace level during PLTGI at phase lags of 90 or $270 \mathrm{deg}$ were approximately the same as the trace level during CGI. The data contained in Figure 2 clearly support the first possibility.

The preceding analysis assumes that the eye was stationary. This assumption may be unwarranted since the subjects were not instructed to maintain eye fixation. However, it was our subjective impression that comparison of the trace motion with respect to the grid was easier if neither the trace nor the grid was tracked. The model required to account for our observations in the moving eye case is complex and the issues raised by this model were not addressed in the present study.

A series of informal observations have been undertaken in which the subjects manipulated the phase angle of PLTGI until the vertical location of the trace relative to the grid appeared the same as during CGI. The results of these informal observations confirmed the results obtained in Experiment 2; the level of the trace during PLTGI at phase lags of 0 and $180 \mathrm{deg}$ appeared to be the same as the level of the trace during CGI. 
In sum, the visual system apparently calculates the position of the trace on a moment-to-moment basis, whereas the grid tends to be seen in its average position during CGI. This difference in determination of grid and trace position by the visual system appears to be the basis for the illusion.

\section{EXPERIMENT 3}

This experiment was designed to demonstrate that the explanation for the illusion supported by Experiment 2 (that the grid tends to be seen in its average position) was not uniquely dependent upon the oscilloscope vertical oscillation rate used in that experiment. Experiment 3 examined the stability of the phase angle at which the level of the trace during PLTGI appeared the same as the level of the trace during CGI across a range of oscilloscope vertical oscillation rates.

\section{Method}

The apparatus, light intensities, shake table displacement, and five of the subjects were the same as those employed in Experiment 2. The procedure for estimating the apparent level of the trace under the conditions of PLTGI and CGI was also the same as in Experiment 2.

Four oscilloscope oscillation rates were examined: $2.5,2.75,3.0$, and $3.25 \mathrm{~Hz}$. Trace velocity was constant at $26.1 \mathrm{~cm} / \mathrm{sec}$. An experimental session consisted of 20 estimates of the level of the trace during PLTGI with respect to the level of the trace during CGI at each of the four trace speeds. The phase angle between PLTGI and periodic oscilloscope displacement was varied randomly prior to each estimate. All 20 estimates were obtained at one trace speed before the speed was changed; however, the order of presentation of the four trace speeds was varied randomly. Experimental sessions took place on five different days. The best-fitting sine function and the phase lag for that function were calculated separately for each block of 20 estimates, as in Figure 2.

\section{Results and Discussion}

The phase lags of the best fitting sine functions exhibited little variation as a function of oscilloscope oscillation rate. Calculated across subjects, the average phase lags of the best fitting functions were as follows: $2.50 \mathrm{~Hz},-9.1 \mathrm{deg} ; 2.75 \mathrm{~Hz},-9.1 \mathrm{deg}$; $3.0 \mathrm{~Hz},-9.3 \mathrm{deg}$; and $3.25 \mathrm{~Hz},-15.6 \mathrm{deg}$. The data obtained in this experiment were evaluated by a two-way analysis of variance. Using days as replicates, significant differences between subjects were noted: $F(4,80)=5.66, p<.01$; however, the difference in phase lags as a function of oscilloscope oscillation rate did not approach significance.

The subjects were different consistently in their reports of the apparent trace levels during the PLTGI relative to the levels during CGI. This observation probably reflects differences in the response criteria employed by the different subjects. Although the results of the analysis of variance indicate significant differences across subjects, perhaps more remarkable is the relatively small range of average phase angles across these subjects --4.4 to $-15.6 \mathrm{deg}$.
The main point to be obtained from these observations is that the phase angles are quite stable. Across a range of table oscillation rates and subjects, the phase angles between PLTGI and oscilloscope displacement at which the apparent level of the trace was the same during CGI and PLTGI remained approximately zero, which suggests that the visual system consistently located the grid at its average displacement position.

\section{GENERAL DISCUSSION}

\section{Observer Motion vs. Target Motion}

The apparent vertical motion of the trace with respect to the grid during vertical oscilloscope oscillation can be comprehended by considering the retinal stimulation, as illustrated in the discussion of Experiment 2 . The retinal stimulation should be similar when the illusion is produced by shaking the oscilloscope or by observing a stationary oscilloscope and stamping your foot. In the former case the stimulus moves vertically across the retina because of motion of the stimulus source, whereas in the latter case the stimulus moves across the retina because of motion of the eye.

It is well known that information processing in the visual system changes when an observer engages in active movements. A complete account of the relationship between illusions produced when the observer is stationary as opposed to those produced when the observer moves may have to take into account some variation of a reafference theory (see Barlow \& Ciganek, 1969).

Two qualitative observations support the position that our illusion can be understood in terms of the analysis provided previously. First, an illusion which is similar to the one produced by shaking the oscilloscope is elicited when the subject's head is oscillated by the same apparatus that was used to drive the shake table. (These experiments were not pursued because of subject discomfort.) Second, several observers have indicated that they are able to partially cancel the apparent motion of the oscilloscope by moving their heads up and down at the same rate as the oscilloscope motion.

\section{Relationship to Other Illusions}

We have noted but not investigated systematically various unusual motions of the trace if we move our eyes during the trace sweep. Undoubtedly similar effects have been noted by nearly everyone who has monitored an oscilloscope. In our experience, these eye-movement-induced illusions are similar to the one described in this paper because the trace appears to move with respect to the grid in a manner that cannot be accounted for by considering the stimulus only.

It has been suggested that the illusion described in this paper is analogous to one considered by 
MacKay (1958). MacKay described illusory displacement of a self-luminous dot with respect to the background when the background was illuminated transiently with a stroboscope. Important differences between MacKay's illusion and the one described in this paper include the following: (a) MacKay's background illumination was discontinuous, whereas the grid in the present illusion was illuminated continuously; and (b) the selfluminous objects did not move with respect to the background in MacKay's illusion, whereas the trace (luminous dot) did move with respect to the grid in the present illusion. The differences between MacKay's illusion and the one described in this paper require postulation of different underlying neural mechanisms. MacKay's illusion supports the notion that different neural channels carry information concerning stimulus motion (brushing) and stimulus position. On the other hand, the present illusion seems to require different processing of the information from the grid and from the trace within the same set of neural channels.

\section{Limitations}

It is clear that the mechanism proposed and examined in Experiment 2 does not account completely for the phenomenon that we have described. The grid is not seen as stationary and in its average position when the oscilloscope is oscillated in the vertical plane. However, partial averaging of vertical grid position would produce the illusion, and our experiments suggest that such a partial averaging may take place within the visual system.

It is difficult to determine the role that perceived direction of grid motion (up and down vs. back and forth) might have in producing the illusion. It is easily understood that the trace should appear to move vertically with respect to the grid if the grid is perceived as moving toward and away from the observer. However, experienced observers report that it is difficult for them to "see" the grid as moving in that fashion; nevertheless, these experienced observers do report the illusion consistently.

\section{Practical Implications}

There are several instances in which pilots or navigators monitor moving traces on displays such as radar screens. If, during these observations, the aircraft encounters turbulence or is otherwise subjected to transient motion, the pilot should observe an illusory displacement of the trace or target blip with respect to the grid or background. If a pilot were attempting to trace a target, this illusion could result in target loss and perhaps even pilot disorientation.

The situation for a pilot in a moving aircraft is more difficult to describe completely than the one considered in this paper, because in the former case both the eye and the target move. It is reasonable to expect that some stimulus motion combinations would result in cancellation of the illusory component of the target motion and other stimulus combinations could exacerbate the illusion. These matters should be investigated.

We have enjoyed the speculation that a few instances of flying saucer sightings might be attributed to the illusion. Consider the case of a person whose eye is transiently displaced while driving along a bumpy road at night. The stimulus situation might be analogous to the one that we have produced in the laboratory. If the sky was clear, the stars might provide a "stationary," grid-like background. Across this background moves an aircraft, visible only as a point of light. As the person's eye is displaced, the aircraft appears to move erratically with respect to the background. For particular individuals, the apparently erratically moving point of light might be reported as a flying saucer.

\section{REFERENCE NOTE}

1. Kabrisky, M. Personal communication, February 1971.

\section{REFERENCES}

Barlow, J. S., \& Ciganex, L. Lambda responses in relation to visual evoked responses in man. Electroencephalography and Clinical Neurophysiology, 1969, 26, 183-192.

Enroth-Cugele, C., \& Robson, J. G. The contrast sensitivity of retinal ganglion cells of the cat. Joumal of Physiology (London), $1966,187,517-552$.

Grusser, O. J., \& Grüsser-Cornehls, H. U. Neuronal mechanisms of visual movement perception and some psychophysical and behavioral correlations. In R. Jung (Ed.), Handbook of sensory physiology (Vol. VII/3). Berlin: Springer-Verlag, 1973.

MAcKAY, D. M. Perceptual stability of a stroboscopically lit visual field containing self-luminous objects. Nature, 1958, 181, 507-508.

(Received for publication June 30, 1976; revision accepted March 16, 1977.) 